\begin{tabular}{|c|c|c|}
\hline 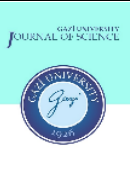 & $\begin{array}{c}\text { Gazi University } \\
\text { Journal of Science }\end{array}$ & 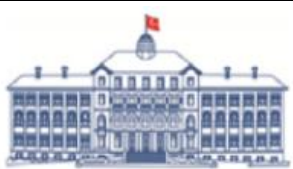 \\
\hline & http://dergipark.gov.tr/gujs & \\
\hline
\end{tabular}

\title{
Bayesian Estimation of the Shape Parameter of Lomax Distribution under Uniform and Jeffery Prior with Engineering Applications
}

\author{
Muhammad IJAZ* \\ Department of Statistics,University of Peshawar, 25120, Peshawar,Pakistan \\ Highlights \\ - This paper focuses on the Bayesian estimation of the Lomax distribution. \\ - The paper delineates the Bayesian estimation under a Uniform and Jeffery priors. \\ - The paper presents a comparison between of Bayesian estimation under various loss functions. \\ - The paper presents a real life application and a simulated data.
}

Article Info

Received: 08 July 2020

Accepted: 23 Oct 2020

Keywords

Lomax distribution

Uniform prior

Jeffery prior

Applications

\begin{abstract}
In engineering, it is usual to model the data so as to make a decision under the problem of uncertainty. Commonly, the data in engineering is skewed to the right, and the skewed distributions in statistics are the appropriate models for making a decision under the Bayesian paradigm. To model the lifetime of an electronic device, an engineer can use the Bayesian estimators to compute the effect of the evidence in increasing the probability for the lifetime of an electronic device by using the prior information. This study presents an estimation of the shape parameter of Lomax distribution under Uniform and Jeffery prior by adopting SELF, QELF, WSELF, and the PELF. The significance of various estimators is compared and presented in graphs using simulated data under the Bayesian paradigm. It was determined that under a uniform prior, Bayes estimator under weighted error loss function (BWEL) provides a better result than others. Under Jeffery prior, the precautionary error loss function (BPEL) leads to a better result than others. Moreover, an application to engineering is also presented for illustration purposes.
\end{abstract}

\section{INTRODUCTION}

The Lomax distribution is one of the most commonly used distribution by the practitioner while modeling the data comes from lifetime experiments. The applications of this distribution are found in engineering, computer science, medical and other related sciences. This distribution was applied by [1-2] to model income and wealth related data, the distribution of computer files on server [3], etc. Many researchers have changed the Lomax distribution, e.g. Ghitany and Al-Awadhi [4] suggested a compound version of the exponentially distributed Lomax distribution. As a baseline distribution, Zografos et.al [5] employed the Cdf of the Lomax distribution. The concept of the Mcdonald-G distribution family with a Lomax baseline feature was demonstrated by Lemonte et.al [6]. Ibrahim et.al [7] adjusted the distribution of Lomax by generating the actual number with the power of the distribution of Cdf of Lomax.

Recently, the Bayesian analysis has got great attention from researchers to analyze the data under the Bayesian paradigm rather than the classical approach. In the statistical inference of the Bayesian approach, one must consider the appropriate prior probability distribution. The prior probability distribution is obtained by taking

* e-mail: ijaz.statistics@gmail.com 
conjugate prior, gamma prior, and Jeffery priors. In order to obtain the best representative value of the unknown parameters of the model, we have to use different loss functions. In this paper, we have considered different loss functions under uniform and Jeffrey prior. The loss function may be symmetric or asymmetric in nature. The symmetric loss functions are includes SELF and the asymmetric loss functions are includes QELF, WELF, and PELF.

The Bayesian analysis of the unknown parameters is studied by many researchers, for example, Hasan and Baizid [8] discussed the Bayesian analysis of the parameter of Exponential distribution, Canavos and Taokas [9] presented the Bayesian analysis of the Weibull distribution. Guure et.al [10] explored the Bayesian estimation of two-parameter Weibull distribution using an extension of Jeffrey's' prior information. Okasha [11] presented the Bayesian estimation of the Lomax distribution using type-II censored data. Nasiri and Hosseini [12] discussed the Bayesian and classical estimation of parameters using record values. Jeffery's [13] worked on invariant form for the prior probability function in estimation problems. Berger et.al [14] discuused the Bayesian anlysis for the Ploy-Weibull distribution, for the Bayesian analysis of other distributions, we refer to see, [15-20].

In this paper, we discussed the Bayesian estimation of the shape parameter of the Lomax distribution under uniform and Jeffery prior by adopting four loss functions. The main goal of the paper is to decide the loss function which would provide a better estimate for the shape parameter using a uniform and Jeffery prior.

A positive continuous random variable $\mathrm{X}$ follows a Lomax distribution with parameter $\vartheta, \lambda>0$ if it has the following Cdf

$$
F(x)=1-\left[1+\left(\frac{x}{\lambda}\right)\right]^{-\vartheta}, x>0 \text { and } \vartheta, \lambda>0 \text {. }
$$

The corresponding probability density function is given by

$$
f(x)=\frac{\vartheta}{\lambda}\left[1+\left(\frac{x}{\lambda}\right)\right]^{-(\vartheta+1)}, x>0
$$

The paper explored the Bayes estimates of the shape parameter $\vartheta$ under uniform and Jeffery priors by using different loss functions.

\section{PRIOR AND POSTERIOR DISTRIBUTION}

To estimate the Bayes estimator, we need to specify the prior probability distribution under uniform and Jeffery prior. The prior distribution for the Lomax distribution under uniform prior is given by

$\pi(\theta) \propto 1$.

Then the posterior distribution of the Lomax distribution is defined as

$$
f\left(\theta / x_{i}\right)=\frac{\prod_{i=1}^{n} f\left(x_{i} / \theta\right) \pi(\theta)}{\int_{i=1}^{n} f\left(x_{i} / \theta\right) f(\theta) d \theta}
$$


where ${ }_{i=1}^{n} f\left(x_{i} / \theta\right)$ is the maximum likelihood function of Lomax distribution and is defined as $\underset{i=1}{n} f\left(x_{i} / \theta\right)$ $=\left(\frac{\vartheta}{\lambda}\right)^{n} \exp \left(-(\vartheta+1) \sum_{i} \log \left[1+\left(\frac{x_{i}}{\lambda}\right)\right]\right), x>0$.

Then (3) becomes

$$
\begin{aligned}
& f\left(\theta / x_{i}\right)=\frac{\left(\frac{\vartheta}{\lambda}\right)^{n} \exp \left(-(\vartheta+1) \sum_{i} \log \left[1+\left(\frac{x_{i}}{\lambda}\right)\right]\right) * 1}{\int\left(\frac{\vartheta}{\lambda}\right)^{n} \exp \left(-(\vartheta+1) \sum_{i} \log \left[1+\left(\frac{x_{i}}{\lambda}\right)\right]\right) * 1 d \theta} \\
& f\left(\theta / x_{i}\right)=\frac{B^{n+1}}{\Gamma(n+1)} \vartheta^{n} \exp (-\vartheta B), \vartheta>0 .
\end{aligned}
$$

This implies that $f\left(\theta / x_{i}\right) \sim \operatorname{Gamma}(\mathrm{n}+1, \mathrm{~B})$.

where, $B=\sum_{i=1}^{n} \log \left[1+\left(\frac{x_{i}}{\lambda}\right)\right]$.

Now the prior distribution for Lomax distribution under Jeffery prior is obtained as follows

$$
\pi(\theta)=\left[-E \frac{\partial^{2} \log L}{\partial \theta^{2}}\right]
$$

The log likelihood function of the Lomax distribution is given by

$\log L\left(x_{i}, \theta\right)=n \log (\vartheta / \lambda)-(\vartheta+1) \sum_{i} \log \left[1+\left(\frac{x_{i}}{\lambda}\right)\right]$.

Differentiating with respect to the parameter $\theta$, we have

$$
\frac{\partial \log L\left(x_{i}, \theta\right)}{\partial \theta}=\frac{n}{\theta}-\sum_{i} \log \left[1+\left(\frac{x_{i}}{\lambda}\right)\right] .
$$

Again, differentiating with respect to the parameter $\theta$, we have

$$
\frac{\partial^{2} \log L\left(x_{i}, \theta\right)}{\partial \theta^{2}}=-\left(\frac{n}{\theta^{2}}\right)^{1 / 2} .
$$

Taking expectation and the square root of the above equation, we determined 


$$
\begin{aligned}
& {\left[-E\left\{\frac{\partial^{2} \log L\left(x_{i}, \theta\right)}{\partial \theta^{2}}\right\}\right]^{\frac{1}{2}}=\left(\frac{n}{\theta^{2}}\right)^{\frac{1}{2}}} \\
& {\left[-E\left\{\frac{\partial^{2} \log L\left(x_{i}, \theta\right)}{\partial \theta^{2}}\right\}\right]^{\frac{1}{2}}=\frac{n^{\frac{1}{2}}}{\theta} .}
\end{aligned}
$$

Finally, we obtained the Jeffery prior as

$$
\pi(\theta)=f(\theta) \propto \theta^{-1} .
$$

Hence, the posterior probability distribution is defined by

$$
f\left(\theta / x_{i}\right)=\frac{\left(\frac{\vartheta}{\lambda}\right)^{n} \exp \left(-(\vartheta+1) \sum_{i} \log \left[1+\left(\frac{x_{i}}{\lambda}\right)\right]\right) * \frac{1}{\vartheta}}{\int\left(\frac{\vartheta}{\lambda}\right)^{n} \exp \left(-(\vartheta+1) \sum_{i} \log \left[1+\left(\frac{x_{i}}{\lambda}\right)\right]\right) * \frac{1}{\vartheta} d \theta} .
$$

Finally, the posterior distribution takes the following form

$$
f\left(\theta / x_{i}\right)=\frac{B^{n}}{\Gamma(n)} \vartheta^{n-1} \exp (-\vartheta B), \vartheta>0
$$

This implies that $f\left(\theta / x_{i}\right) \sim \operatorname{Gamma}(\mathrm{n}, \mathrm{B})$.

\section{BAYESIAN ESTIMATORS UNDER UNIFORM PRIOR USING DIFFERENT LOSS FUNCTIONS}

In this section, we have derived Bayes estimators of the parameter $\theta$ under uniform prior to using various loss functions.

\subsection{Squared Error Loss Function}

The Bayes estimator of $\theta$ under SELF [14] function is defined by

$L(\hat{\theta}, \theta)=(\hat{\theta}-\theta)^{2}$

Using the above loss function, the Bayes estimator of $\theta$ is defined by solving the equation

$$
\begin{gathered}
\frac{\partial}{\partial \hat{\theta}} \int(\hat{\theta}-\theta)^{2} f(\theta / x) d \theta=0 \\
\int \hat{\theta} f(\theta / x) d \theta-\theta \int f(\theta / x)=0
\end{gathered}
$$




$$
\begin{aligned}
& \frac{B^{n+1}}{\Gamma(n+1)} \hat{\theta} \int \vartheta^{n} \exp (-\vartheta B) d \theta-\frac{B^{n+1}}{\Gamma(n+1)} \int \vartheta^{n+1} \exp (-\vartheta B) d \theta=0 \\
& \frac{B}{(n)} \hat{\theta}-\frac{(n+1)}{B}=0 .
\end{aligned}
$$

Finally, we determined the result for $\hat{\theta}$

$\hat{\theta}_{S E L F}=\frac{n(n+1)}{B^{2}}$.

\subsection{Quadratic Loss Function}

The QELF [15] is defined by

$L(\hat{\theta}, \theta)=\left(\frac{\hat{\theta}-\theta}{\theta}\right)^{2}$

The estimator $\hat{\theta}$ is defined by solving the equation

$$
\frac{\partial \int L(\hat{\theta}, \theta)}{\partial \hat{\theta}}=0
$$

$\frac{\partial}{\partial \hat{\theta}} \int\left(\frac{\hat{\theta}-\theta}{\theta}\right)^{2} f(\theta / x) d \theta=0$

$\int \frac{\hat{\theta}}{\theta^{2}} f(\theta / x) d \theta-\int \frac{\theta}{\theta^{2}} f(\theta / x) d \theta=0$

$\frac{B^{n+1}}{\Gamma(n+1)}\left[\hat{\theta} \int \vartheta^{n-2} \exp (-\vartheta B) d \theta-\int \vartheta^{n-1} \exp (-\vartheta B) d \theta\right]=0$

$\hat{\theta} \frac{\Gamma(n-1)}{B^{n-1}}-\frac{\Gamma n}{B^{n}}=0$.

Finally, we determined the following result

$$
\hat{\theta}_{Q E L F}=\frac{(n-1)}{B} .
$$

\subsection{Weighted Square Loss Function}

The WELF is defined by 
$L(\hat{\theta}, \theta)=\frac{(\hat{\theta}-\theta)}{\theta}$

The estimator of $\hat{\theta}$ under the WELF is defined by the equation

$\frac{\partial}{\partial \hat{\theta}} \int L(\hat{\theta}, \theta) d \theta=0$

$\frac{\partial}{\partial \hat{\theta}} \int \frac{(\hat{\theta}-\theta)^{2}}{\theta} f(\theta / x) d \theta=0$

$\int \frac{\hat{\theta}}{\theta} f(\theta / x) d \theta-\frac{\theta}{\theta} \int f(\theta / x) d \theta=0$

$\frac{B^{n+1}}{\Gamma(n+1)}\left[\hat{\theta} \int \vartheta^{n-1} \exp (-\vartheta B) d \theta-\int \vartheta^{n+1-1} \exp (-\vartheta B) d \theta\right]=0$

$\hat{\theta} \frac{\Gamma(n)}{B^{n}}-\frac{\Gamma(n+1)}{B^{n+1}}=0$.

Finally, we get the following result

$\hat{\theta}_{W E L F}=\frac{(n)}{B}$.

\subsection{Precautionary Loss Function}

The PELF is given by

$L(\hat{\theta}, \theta)=\frac{(\hat{\theta}-\theta)^{2}}{\hat{\theta}}$.

The Bayes estimator $\hat{\theta}$ PELF is defined by the equation

$\frac{\partial}{\partial \hat{\theta}} \int L(\hat{\theta}, \theta)=0$

$\frac{\partial}{\partial \hat{\theta}} \int \frac{(\hat{\theta}-\theta)^{2} \theta^{n-1}\left(\sum x_{i}\right)^{n} \mathrm{e}^{-\theta \sum x_{i}}}{\hat{\theta}) n} d \theta=0$

$\frac{\partial}{\partial \hat{\theta}} \int \frac{\hat{\theta}^{2}-\theta^{2}}{\hat{\theta}^{2}} \frac{\left(\sum x_{i}\right)^{n}}{\sqrt{n}} \theta^{n-1} \mathrm{e}^{-\theta \sum x_{i}} d \theta=0$ 


$$
\begin{aligned}
& \frac{B^{n+1}}{\Gamma(n+1)}\left[\int \vartheta^{n} \exp (-\vartheta B) d \theta-\frac{1}{\hat{\theta}^{2}} \int \vartheta^{n+3-1} \exp (-\vartheta B) d \theta\right]=0 \\
& \frac{\Gamma(n)}{B^{n}}-\frac{1}{\hat{\theta}^{2}} \frac{\Gamma(n+3)}{B^{n+3}}=0 .
\end{aligned}
$$

Hence, we determined the result

$$
\hat{\theta}_{P L F}=\sqrt{\frac{n(n+1)(n+2)}{B^{3}}} .
$$

\section{BAYESIAN ESTIMATORS UNDER JEFFERY PRIOR USING DIFFERENT LOSS FUNCTIONS}

In this section, we derived Bayes estimators of the parameter $\theta$ under Jeffery prior by using various loss functions.

\subsection{Squared Error Loss Function}

The Bayes estimator of $\theta$ under SELF [14] function is defined by

$L(\hat{\theta}, \theta)=(\hat{\theta}-\theta)^{2}$

The Bayes estimator of $\theta$ is defined by solving the equation

$$
\begin{aligned}
& \frac{\partial}{\partial \hat{\theta}} \int(\hat{\theta}-\theta)^{2} f(\theta / x) d \theta=0 \\
& \int \hat{\theta} f(\theta / x) d \theta-\theta \int f(\theta / x)=0 \\
& \frac{B^{n}}{\Gamma(n)}\left[\hat{\theta} \int \vartheta^{n-1} \exp (-\vartheta B) d \theta-\int \vartheta^{n-1+1} \exp (-\vartheta B) d \theta\right]=0 \\
& \frac{\Gamma n}{B^{n}} \hat{\theta}-\frac{\Gamma(n+1)}{B^{n+1}}=0 .
\end{aligned}
$$

Finally, we determined the result for $\hat{\theta}$

$$
\hat{\theta}_{S E L F}=\frac{n}{B}
$$

\subsection{Quadratic Loss Function}

The QELD [15] is defined by 
$L(\hat{\theta}, \theta)=\left(\frac{\hat{\theta}-\theta}{\theta}\right)^{2}$

The estimator $\hat{\theta}$ is defined by solving the equation

$$
\frac{\partial \int L(\hat{\theta}, \theta)}{\partial \hat{\theta}}=0
$$

$\frac{\partial}{\partial \hat{\theta}} \int\left(\frac{\hat{\theta}-\theta}{\theta}\right)^{2} f(\theta / x) d \theta=0$

$\int \frac{\hat{\theta}}{\theta^{2}} f(\theta / x) d \theta-\int \frac{\theta}{\theta^{2}} f(\theta / x) d \theta=0$

$\frac{B^{n}}{\Gamma(n)}\left[\hat{\theta} \int \vartheta^{n-2-1} \exp (-\vartheta B) d \theta-\int \vartheta^{n-1-1} \exp (-\vartheta B) d \theta\right]=0$

$\hat{\theta} \frac{\Gamma(n-2)}{B^{n-2}}-\frac{\Gamma(n-1)}{B^{n-1}}=0$.

Finally, we determined the following result

$\hat{\theta}_{Q E L F}=\frac{(n-2)}{B}$.

\subsection{Weighted Square Loss Function}

The WELF is defined by

$L(\hat{\theta}, \theta)=\frac{(\hat{\theta}-\theta)}{\theta}$.

The estimator of $\hat{\theta}$ under the WELF is defined by the equation

$$
\begin{aligned}
& \frac{\partial}{\partial \hat{\theta}} \int L(\hat{\theta}, \theta) d \theta=0 \\
& \frac{\partial}{\partial \hat{\theta}} \int \frac{(\hat{\theta}-\theta)^{2}}{\theta} f(\theta / x) d \theta=0 \\
& \int \frac{\hat{\theta}}{\theta} f(\theta / x) d \theta-\frac{\theta}{\theta} \int f(\theta / x) d \theta=0
\end{aligned}
$$




$$
\begin{aligned}
& \frac{B^{n}}{\Gamma(n)}\left[\hat{\theta} \int \vartheta^{n-1-1} \exp (-\vartheta B) d \theta-\int \vartheta^{n-1} \exp (-\vartheta B) d \theta\right]=0 \\
& \hat{\theta} \frac{\Gamma(n-1)}{B^{n-1}}-\frac{\Gamma(n)}{B^{n}}=0 .
\end{aligned}
$$

Finally, we get the following result

$\hat{\theta}_{W E L F}=\frac{(n-1)}{B}$.

\subsection{Precautionary Loss Function}

The PELF is given by

$L(\hat{\theta}, \theta)=\frac{(\hat{\theta}-\theta)^{2}}{\hat{\theta}}$.

The Bayes estimator $\hat{\theta}$ under PELF is defined by the equation

$\frac{\partial}{\partial \hat{\theta}} \int L(\hat{\theta}, \theta)=0$

$\frac{\partial}{\partial \hat{\theta}} \int \frac{(\hat{\theta}-\theta)^{2} \theta^{n-1}\left(\sum x_{i}\right)^{n} \mathrm{e}^{-\theta \sum x_{i}}}{\hat{\theta} \sqrt{n}} d \theta=0$

$\frac{\partial}{\partial \hat{\theta}} \int \frac{\hat{\theta}^{2}-\theta^{2}}{\hat{\theta}^{2}} \frac{\left(\sum x_{i}\right)^{n}}{\sqrt{n}} \theta^{n-1} \mathrm{e}^{-\theta \sum x_{i}} d \theta=0$

$\frac{B^{n}}{\Gamma(n)}\left[\int \vartheta^{n-1} \exp (-\vartheta B) d \theta-\frac{1}{\hat{\theta}^{2}} \int \vartheta^{n+2-1} \exp (-\vartheta B) d \theta\right]=0$

$\frac{\Gamma(n)}{B^{n}}-\frac{1}{\hat{\theta}^{2}} \frac{\Gamma(n+2)}{B^{n+2}}=0$.

Hence, we determined the result

$\hat{\theta}_{P L F}=\frac{\sqrt{n(n+1)}}{B}$

\section{QUANTILE FUNCTION}

To generate random data from the Lomax distribution, we need to use the quantile function of the Lomax distribution. Let $\mathrm{X}$ is a Lomax random variable, then, the quantile function is defined by 
$F(x)=u$,

$F(x)=1-\left[1+\left(\frac{x}{\lambda}\right)\right]^{-\vartheta}, x>0$ and $\vartheta, \lambda>0$

by the inverse transformation we have,

$u=1-\left[1+\left(\frac{x}{\lambda}\right)\right]^{-\vartheta}$

When we solve the above equation for $x$, we obtained the result

$x=\frac{\lambda}{(1-u)^{1 / \vartheta}}$

where, $U$ is uniformly distributed over the range [0-1].

\section{EMPIRICAL ANALYSIS}

In this study, using Monte Carlo simulation method we have generated a sample of size 6000 for each estimator in case of uniform and Jeffery prior. We have computed the estimated values of $\hat{\theta}_{S L F} \hat{\theta}_{Q L}, \hat{\theta}_{W S E L}, \hat{\theta}_{P L F}$. For comparison, we have considered the mean squared error (MSE) of the estimator which is defined by

$\operatorname{MSE}(\hat{\theta})=E(\hat{\theta}-\theta)^{2}=\operatorname{var}(\hat{\theta})+[\operatorname{Biass}(\hat{\theta})]^{2}$

A short algorithm for the Monte Carlo simulation from the Lomax distribution is given below;

i. Generate samples from the Lomax distribution by using the quantile function $x=\frac{\lambda}{(1-u)^{1 / \vartheta}}$, where $\mathrm{U}$ is the standard uniform random variable.

ii. Obtain Bayes estimator of various loss functions under uniform and Jeffery prior.

iii. The above steps are repeated 6000 times for each sample size and obtained the estimated values of Bayes estimators and their MSE.

The results of estimated values and MSE of Bayes estimators under uniform and Jeffery prior are given in the following tables.

Table 1. Estimated value and MSE of $\theta$ under uniform Prior, when $n=30$ and $\theta=0.5$.

\begin{tabular}{|c|c|c|c|c|c|}
\hline$\lambda$ & criteria & BSE & BQEL & BWEL & BPEL \\
\hline 1 & Estimated value & 0.2149213 & 0.4370818 & 0.4508266 & 0.3203866 \\
& MSE & 0.08545983 & 0.008298509 & 0.00687226 & 0.03741835 \\
\hline 1.3 & Estimated value & 0.2164128 & 0.4376607 & 0.4497425 & 0.3225422 \\
& MSE & 0.08483227 & 0.008240077 & 0.006887342 & 0.03688659 \\
\hline 1.5 & Estimated value & 0.2152392 & 0.435493 & 0.4522239 & 0.3218992 \\
\hline
\end{tabular}




\begin{tabular}{|c|c|c|c|c|c|}
\hline & MSE & 0.08525462 & 0.008274607 & 0.00681566 & 0.03709663 \\
\hline 1.7 & Estimated value & 0.2152392 & 0.4357685 & 0.4516097 & 0.3200244 \\
& MSE & 0.08525462 & 0.008225762 & 0.006823928 & 0.03746877 \\
\hline 1.9 & Estimated value & 0.2157496 & 0.4379354 & 0.4519274 & 0.3203169 \\
& MSE & 0.08507986 & 0.008081702 & 0.006833053 & 0.03735006 \\
\hline 2 & Estimated value & 0.2151436 & 0.435133 & 0.4514845 & 0.3227669 \\
& MSE & 0.08533011 & 0.008228344 & 0.006777749 & 0.03664439 \\
\hline 2.3 & Estimated value & 0.2152008 & 0.435933 & 0.4522258 & 0.3195773 \\
& MSE & 0.08533154 & 0.008354807 & 0.006675974 & 0.03749064 \\
\hline 2.5 & Estimated value & 0.2148919 & 0.4358239 & 0.4513706 & 0.3205905 \\
& MSE & 0.08541602 & 0.008242673 & 0.006867066 & 0.03743278 \\
\hline
\end{tabular}

Table 2. Estimated value and MSE of $\theta$ under uniform Prior, when $\theta=0.5$ and $\lambda=0.3$

\begin{tabular}{|c|c|c|c|c|c|}
\hline $\mathrm{n}$ & criteria & BSE & BQEL & BWEL & BPEL \\
\hline 5 & Estimated value & 0.3319291 & 0.3999805 & 0.5020937 & 0.4783227 \\
& MSE & 0.08568496 & 0.03017299 & 0.03040638 & 0.06517857 \\
\hline 10 & Estimated value & 0.2587462 & 0.4206621 & 0.4704327 & 0.3785812 \\
& MSE & 0.07672004 & 0.01762761 & 0.01527385 & 0.03501358 \\
\hline 15 & Estimated value & 0.2390543 & 0.4307223 & 0.4597632 & 0.3500939 \\
& MSE & 0.07852516 & 0.01267017 & 0.01103127 & 0.03491594 \\
\hline 20 & Estimated value & 0.2248752 & 0.4335792 & 0.4567135 & 0.3344758 \\
& MSE & 0.08234094 & 0.01068689 & 0.008875785 & 0.03589976 \\
\hline 25 & Estimated value & 0.2201417 & 0.4355578 & 0.4539721 & 0.3274998 \\
& MSE & 0.08353796 & 0.009301464 & 0.007530034 & 0.03631128 \\
\hline 30 & Estimated value & 0.2155449 & 0.4361713 & 0.4506884 & 0.3216516 \\
& MSE & 0.08510238 & 0.008307794 & 0.006929624 & 0.03703072 \\
\hline 35 & Estimated value & 0.2120455 & 0.4386988 & 0.4491208 & 0.3172439 \\
& MSE & 0.08629403 & 0.007355766 & 0.006427789 & 0.03781508 \\
\hline
\end{tabular}

In Table 1 , for a fixed value of $\mathrm{n}=30$ and different values of the scale parameter, In Table 2 , for $\theta=0.5$ and $\lambda=0.3$, we estimated the shape parameter for different sample sizes. Tables 1 and 2 clearly show that the MSE of the Bayes estimator under precautionary loss function is very high among other estimators but as we increase the sample size, their MSE decreases continuously and become closer to the MSE of other estimators. However, the MSE under BWEL is minimum among other estimators in both Tables 1 and 2. Furthermore, as we increase the sample size, the MSE of the shape parameter of Lomax distribution under BQEL and PELF becomes identical as shown in Figure 1. 


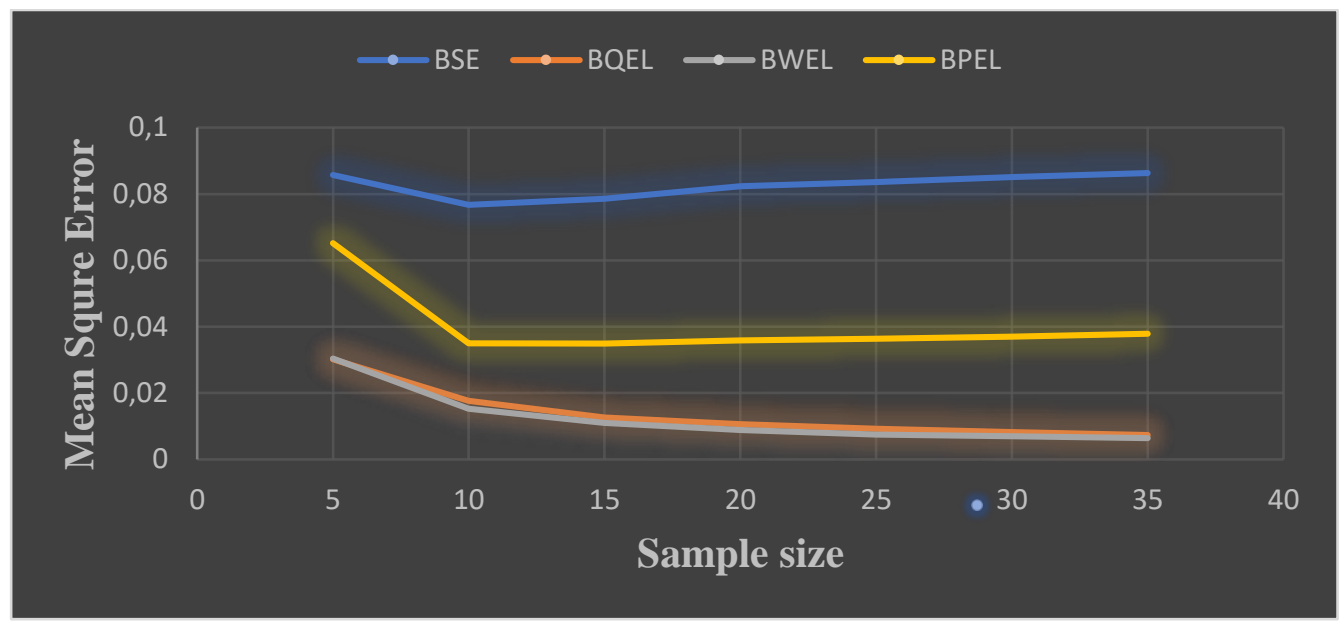

Figure 1. Graph of MSE for Different estimators when $\theta=0.5$ and $\lambda=0.3$

Table 3. Estimated value and MSE of $\theta$ under Jeffery Prior, when $n=30$ and $\theta=0.5$

\begin{tabular}{|l|l|l|l|l|l|}
\hline$\lambda$ & criteria & BSE & BQEL & BWEL & BPEL \\
\hline 1 & Estimated value & 0.4519052 & 0.4220284 & 0.4362834 & 0.4575921 \\
& MSE & 0.006923581 & 0.009905471 & 0.008242466 & 0.006305317 \\
\hline 1.3 & Estimated value & 0.4505209 & 0.4201514 & 0.4361401 & 0.4588445 \\
& MSE & 0.00686885 & 0.01034187 & 0.00829987 & 0.006142034 \\
\hline 1.5 & Estimated value & 0.4506821 & 0.4219039 & 0.4351151 & 0.4596623 \\
& MSE & 0.006883 & 0.009993571 & 0.008376783 & 0.006359995 \\
\hline 1.7 & Estimated value & 0.4512308 & 0.4220043 & 0.4377546 & 0.4585454 \\
& MSE & 0.006750326 & 0.009938195 & 0.00814634 & 0.006367157 \\
\hline 1.9 & Estimated value & 0.4517657 & 0.4203534 & 0.4363578 & 0.4596716 \\
& MSE & 0.006972674 & 0.01013376 & 0.008316073 & 0.00641334 \\
\hline 2 & Estimated value & 0.4512041 & 0.4214374 & 0.436754 & 0.4585939 \\
& MSE & 0.006810138 & 0.0100154 & 0.008255356 & 0.006397397 \\
\hline 2.3 & Estimated value & 0.4515365 & 0.4219088 & 0.4372719 & 0.4592931 \\
& MSE & 0.006796453 & 0.009981116 & 0.008193196 & 0.006484479 \\
\hline 2.5 & Estimated value & 0.4503184 & 0.420306 & 0.4371422 & 0.4589022 \\
& MSE & 0.007066036 & 0.01031897 & 0.008047473 & 0.006396922 \\
\hline
\end{tabular}

Table 4. Estimated value and MSE of $\theta$ under Jeffery Prior, when $\theta=0.5$ and $\lambda=2.5$

\begin{tabular}{|l|l|l|l|l|l|}
\hline $\mathrm{n}$ & criteria & BSEL & BQEL & BWEL & BPEL \\
\hline 20 & Estimated value & 0.4562568 & 0.4113107 & 0.4335889 & 0.4668433 \\
& MSE & 0.00880651 & 0.01341047 & 0.01044819 & 0.008288899 \\
\hline 25 & Estimated value & 0.454191 & 0.4168556 & 0.4366963 & 0.4618986 \\
& MSE & 0.007522211 & 0.01142017 & 0.009030617 & 0.007038851 \\
\hline \multirow{2}{3}{30} & Estimated value & 0.4507528 & 0.4213435 & 0.4354045 & 0.458959 \\
& MSE & 0.007006199 & 0.0101068 & 0.008307336 & 0.006257292 \\
\hline 35 & Estimated value & 0.4508849 & 0.4243753 & 0.4369676 & 0.4577372 \\
& MSE & 0.006263382 & 0.009060677 & 0.007580084 & 0.00578842 \\
\hline
\end{tabular}




\begin{tabular}{|l|l|l|l|l|l|}
\hline 40 & Estimated value & 0.4479669 & 0.4277797 & 0.4384899 & 0.4540659 \\
& MSE & 0.005902645 & 0.008239798 & 0.006990802 & 0.005498376 \\
\hline 45 & Estimated value & 0.4485961 & 0.4282016 & 0.437944 & 0.4539746 \\
& MSE & 0.005642649 & 0.007899972 & 0.006676798 & 0.005127907 \\
\hline 50 & Estimated value & 0.4485166 & 0.4287726 & 0.4383064 & 0.4519187 \\
& MSE & 0.005642649 & 0.007506829 & 0.006281222 & 0.005018414 \\
\hline
\end{tabular}

In Table 3, the shape parameter is estimated using different loss functions with various values of $\lambda$. It has been observed that for fixed values of $\mathrm{n}$ and scale parameter, BPEL provides a better result as compared to others. Table 4 shows that by increasing the sample size; the MSE are decreases and it is found that the MSE of BPEL is minimum among others. Moreover, Table 4 clearly shows that as we increase the sample size, BSE and BPEL rapidly becomes identical as shown in Figure 2.

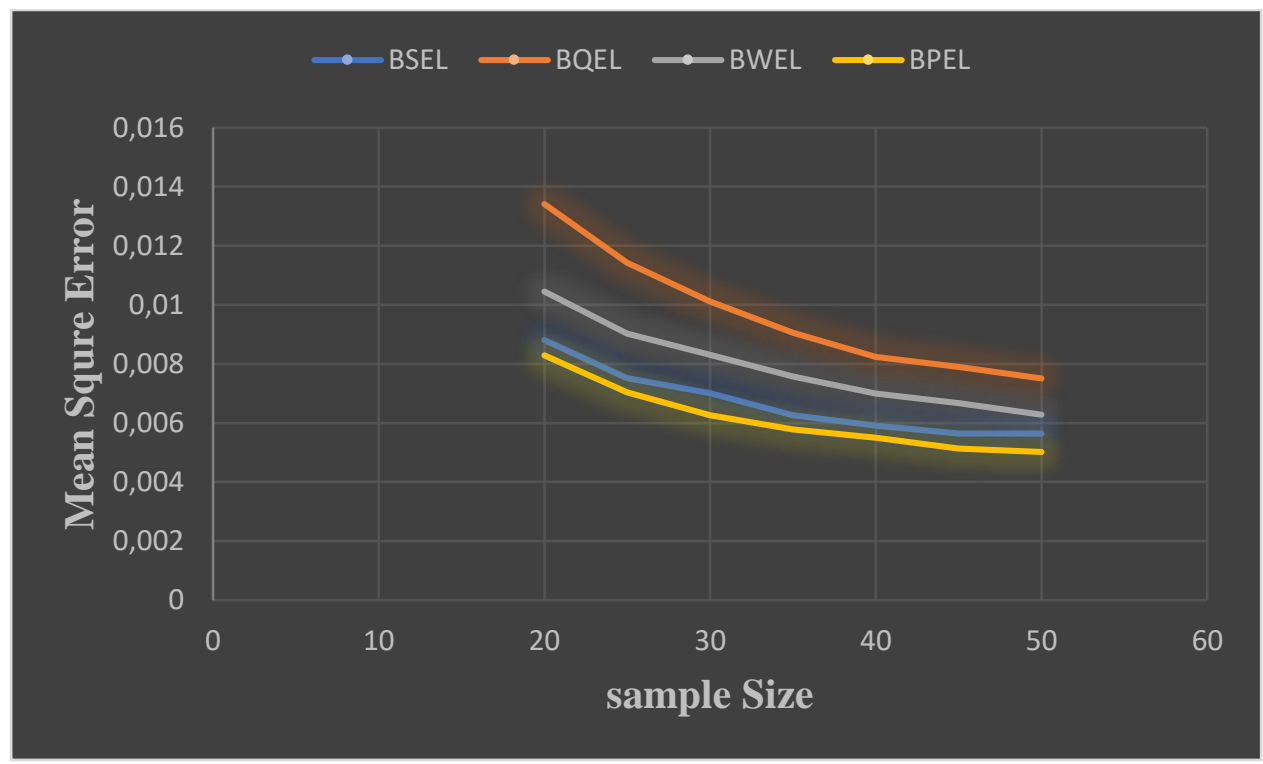

Figure 2. Graph of MSE of different estimators of when $\theta=0.5$ and $\lambda=2.5$

\section{APPLICATION}

For the purpose of illustration, Arset data has been considered which has been cited by Almalki and Nadarajah in 2014 [21]. The data set represent the failure times of 50 electronic devices in weeks. This data set has also been cited by El-Morshedy et.al [22]. The values are as follows 1.7,2.2,14.4,1.1,0.4,20.6,5.3,0.7,1.9,13.0,12.0,9.3,1.4,18.7,8.5,25.5,11.6,14.1,22.1,1.1,2.5,14.4,1.7,37.6,0.6,2. 2,39.0,0.3,15.0,11.0,7.3,22.9,1.7,0.1,1.1,0.6,9.0,1.7,7.0,20.1,0.4,2.8,14.1,9.9,10.4,10.7,30.0,3.6,5.6,30.8,13.3, $4.2,5.5,3.4,11.9,21.5,27.6,36.4,2.7,64.0,1.5,2.5,27.4,1.0,27.1,20.2,16.8,53,9.7,27.5,2.5,27.0$.

Figure 3 demonstrates the histogram and theoretical density of the Aarset data. The graph clearly indicates that the data is stretched out to the right and follows a positively skewed distribution. 


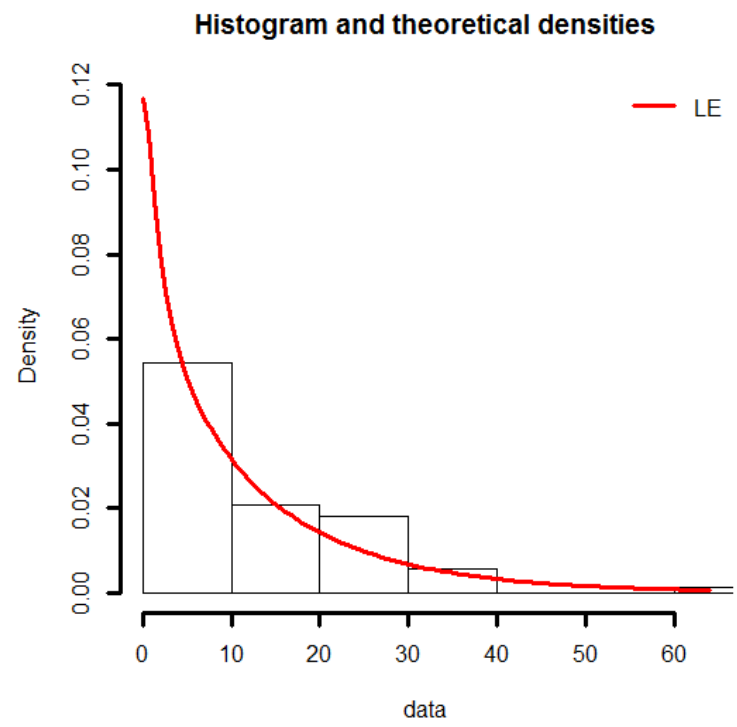

Figure 3. Histogram and Theoretical Density of Electronic Devices Data

Table 5. Estimated value and MSE of $\theta$ under Uniform Prior, when $\theta=0.5$ and $\lambda=0.3$

\begin{tabular}{|c|l|l|l|l|l|}
\hline $\mathrm{n}$ & criteria & BSE & BQEL & BWEL & BPEL \\
\hline 10 & Estimated value & 0.1183661 & 0.1465849 & 0.3233719 & 0.2142106 \\
& MSE & 0.1465849 & 0.04452286 & 0.03279822 & 0.08338582 \\
\hline 20 & Estimated value & 0.1094385 & 0.3057583 & 0.3213516 & 0.1961859 \\
& MSE & 0.1528419 & 0.0383101 & 0.03256214 & 0.09284459 \\
\hline 30 & Estimated value & 0.1062077 & 0.3097262 & 0.3203286 & 0.1907615 \\
& MSE & 0.1552159 & 0.03651601 & 0.03260884 & 0.09589744 \\
\hline 40 & Estimated value & 0.1051924 & 0.3119378 & 0.3200947 & 0.1878566 \\
& MSE & 0.1559533 & 0.03554341 & 0.03256003 & 0.09757849 \\
\hline
\end{tabular}

Table 6. Estimated value and MSE of $\theta$ under Jeffery Prior, when $\theta=0.5$ and $\lambda=0.5$

\begin{tabular}{|c|l|l|l|l|l|}
\hline $\mathrm{n}$ & criteria & BSE & BQEL & BWEL & BPEL \\
\hline 10 & Estimated value & 0.3789634 & 0.3033679 & 0.3426587 & 0.3991778 \\
& MSE & 0.01747537 & 0.04036066 & 0.0271017 & 0.01319617 \\
\hline 20 & Estimated value & 0.3760454 & 0.3384607 & 0.3566594 & 0.3848476 \\
& MSE & 0.0164588 & 0.02699666 & 0.0215152 & 0.01437308 \\
\hline \multirow{2}{*}{30} & Estimated value & 0.3742253 & 0.3491387 & 0.3623125 & 0.3810743 \\
& MSE & 0.0163705 & 0.0232366 & 0.01948252 & 0.01470958 \\
\hline 40 & Estimated value & 0.3741812 & 0.3550246 & 0.3646122 & 0.3784653 \\
& MSE & 0.01615611 & 0.02128928 & 0.01862929 & 0.01510511 \\
\hline
\end{tabular}

Again by using real data set, it has been observed that BWEL perform better than other loss functions under a uniform prior, the result is given in Table 5. Moreover, Table 6 reflects Bayesian estimation under Jeffery prior and it is declared that BPEL leads to a better result than other loss functions. 


\section{CONCLUSION}

The paper presents a Bayes estimation of parameter $\theta$ using a Uniform and Jeffery prior under Square error loss function (BSEL), quadratic error loss function (BQEL), weighted error loss function (BWEL), and precautionary error loss function (BPEL). Based on the numerical analysis and graphical representation, it has been determined that under uniform prior, Bayes estimator under weighted error loss function (BWEL) provides a better result than others. Under Jeffery prior, precautionary error loss function (BPEL) leads to a better result than others.

\section{CONFLICTS OF INTEREST}

No conflict of interest was declared by the author.

\section{REFERENCES}

[1] Harris, C. M., "The Pareto distribution as a queue service discipline”, Operations Research, 16(2): 307-313, (1968).

[2] Atkinson, A.B., Harrison, A.J., "Distribution of Personal Wealth in Britain Cambridge University Press", Cambridge, (1978).

[3] Hassan, A.S., Al-Ghamdi, A.S., "Optimum step stress accelerated life testing for Lomax distribution", Journal of Applied Sciences Research, 5: 2153-2164, (2009).

[4] Ghitany, M. E., Al-Awadhi, F. A., Alkhalfan, L. A., "Marshall-Olkin extended Lomax- distribution and its application to censored data", Communications in Statistics, Theory and Methods, 36: 18551866, (2007).

[5] Zografos, K., Balakrishnan, N., "On families of beta-and generalized gamma-generated distributions and associated inference", Statistical Methodology, 6(4): 344-362, (2009).

[6] Lemonte, A. J., Gauss M. C., “An extended Lomax distribution”, Statistics, 47(4): 800-816, (2013).

[7] Ibrahim, A.B., Moniem A., Hameed A., "Exponentiated Lomax distribution", International Journal of Mathematical Education, 33(5): 1-7,( 2012).

[8] Hasan, M. R., Baizid, A. R., "Bayesian Estimation under Different loss Functions Using Gamma Prior for the Case of Exponential Distribution”, Journal of Scientific Research, 9(1): 67-78, (2017).

[9] Canavos, G. C., Taokas, C. P., "Bayesian estimation of life parameters in the Weibull distribution”, Operations Research, 21(3): 755-763, (1973).

[10] Guure, C. B., Ibrahim, N. A., Ahmed, A. O. M., "Bayesian estimation of two-parameter weibull distribution using extension of Jeffreys' prior information with three loss functions", Mathematical Problems in Engineering, (2012). 
[11] Okasha, H. M., "E-Bayesian estimation for the Lomax distribution based on type-II censored data", Journal of the Egyptian Mathematical Society, 22(3): 489-495, (2014).

[12] Nasiri, P., Hosseini, S.,"Statistical inferences for Lomax distribution based on record values (Bayesian and classical)”, Journal of Modern Applied Statistical Methods, 11(1): 15, (2012).

[13] Jeffreys, H., "An invariant form for the prior probability in estimation problems", Proceedings of the Royal Society of London. Series A. Mathematical and Physical Sciences, 186(1007): 453-461, (1946).

[14] Berger, J. O. and Sun, D., "Bayesian analysis for the poly-Weibull distribution." Journal of the American Statistical Association, 88 (424): 1412-1418, (1993).

[15] Naji, Loaiy F., and Huda A. Rasheed. "Bayesian Estimation for Two Parameters of Gamma Distribution under Generalized Weighted loss Function." Iraqi Journal of Science, 60(5): 1161-1171, (2019).

[16] Naji, L. F. and Rasheed, H. A., "Bayesian estimation for two parameters of Gamma distribution under precautionary loss function." Ibn AL-Haitham Journal For Pure and Applied Science, 32(1): 187-196, (2019).

[17] Ni, S. and Sun, D., "Intrinsic Bayesian estimation of linear time series models." Statistical Theory and Related Fields, 1(13), (2020).

[18] Annan, J. D., "Recent developments in Bayesian estimation of climate sensitivity." Current Climate Change Reports, 1(4), 263-267, (2015).

[19] Yadav, A. S., Singh, S. K. and Singh, U., "Bayesian estimation of R=P[Y<X] for inverse Lomax distribution under progressive type-II censoring scheme." International Journal of System Assurance Engineering and Management, 10(5), 905-917, (2019).

[20] Smith, R. L. and Naylor, J. C., "A comparison of maximum likelihood and Bayesian estimators for the three parameter Weibull distribution." Journal of the Royal Statistical Society: Series C (Applied Statistics), 36(3),358-369, (1987).

[21] Almalki, S. J. and Nadarajah, S., "Modifications of the Weibull distribution: A review." Reliability Engineering \& System Safety, 124, 32-55, (2014).

[22] El-Morshedy, M., El-Bassiouny, A. H., El-Gohary, A., "Exponentiated inverse flexible Weibull extension distribution", J. Stat. Appl. Probability, 6(1): 169-83, (2017). 